\title{
Refinements and Sharpening of some Huygens and Wilker Type Inequalities
}

\author{
Wei-Dong Jiang ${ }^{1}$, Qiu-Ming Luo $^{2}$, Feng $\mathbf{Q i}^{3,4,5, *}$ \\ ${ }^{1}$ Department of Information Engineering, Weihai Vocational University, Weihai, Shandong, China \\ ${ }^{2}$ Department of Mathematics, Chongqing Normal University, Chongqing City, China \\ ${ }^{3}$ College of Mathematics, Inner Mongolia University for Nationalities, Tongliao City, Inner Mongolia Autonomous Region, China \\ ${ }^{4}$ Department of Mathematics, College of Science, Tianjin Polytechnic University, Tianjin City, China \\ ${ }^{5}$ Institute of Mathematics, Henan Polytechnic University, Jiaozuo City, Henan Province, China \\ *Corresponding author: qifeng618@gmail.com, qifeng618@hotmail.com, qifeng618@qq.com
}

Received June 30, 2014; Revised August 09, 2014; Accepted August 21, 2014

\begin{abstract}
In the article, some Huygens and Wilker type inequalities involving trigonometric and hyperbolic functions are refined and sharpened.
\end{abstract}

Keywords: refinement, sharpening, Huygens inequality, Wilker inequality, trigonometric function, hyperbolic function

Cite This Article: Wei-Dong Jiang, Qiu-Ming Luo, and Feng Qi, "Refinements and Sharpening of some Huygens and Wilker Type Inequalities." Turkish Journal of Analysis and Number Theory, vol. 2, no. 4 (2014): 134-139. doi: 10.12691/tjant-2-4-6.

\section{Introduction}

The famous Huygens inequality for the sine and tangent functions states that for $x \in\left(0, \frac{\pi}{2}\right)$

$$
2 \sin x+\tan x>3 x .
$$

The hyperbolic counterpart of (1.1) was established in [10] as follows: For $x>0$

$$
2 \sinh x+\tanh x>3 x .
$$

The inequalities (1.1) and (1.2) were respectively refined in [[10], Theorem 2.6] as

$$
2 \frac{\sin x}{x}+\frac{\tan x}{x}>2 \frac{x}{\sin x}+\frac{x}{\tan x}>3
$$

for $0<x<\frac{\pi}{2}$ and

$$
2 \frac{\sinh x}{x}+\frac{\tanh x}{x}>2 \frac{x}{\sinh x}+\frac{x}{\tanh x}>3, x \neq 0 .
$$

In [8] the inequality (1.2) was improved as

$$
2 \frac{\sinh x}{x}+\frac{\tanh x}{x}>3+\frac{3}{20} x^{4}-\frac{3}{56} x^{6}, x>0 .
$$

In [10], the following inequality is given

$$
3 \frac{x}{\sin x}+\cos x>4 \text {. }
$$

For more information in this area, please refer to $[13,16]$, [[14], Section 1.7 and Section 7.3] and closely related references therein.

\section{In [18], Wilker proved}

$$
\left(\frac{\sin x}{x}\right)^{2}+\frac{\tan x}{x}>2
$$

and proposed that there exists a largest constant $c$ such that

$$
\left(\frac{\sin x}{x}\right)^{2}+\frac{\tan x}{x}>2+c x^{3} \tan x
$$

for $0<x<\frac{\pi}{2}$. In [17], the best constant $c$ in (1.7) was found and it was proved that

$$
2+\frac{8}{45} x^{3} \tan x>\left(\frac{\sin x}{x}\right)^{2}+\frac{\tan x}{x}>2+\left(\frac{2}{\pi}\right)^{4} x^{3} \tan x
$$

for $0<x<\frac{\pi}{2}$. The constants $\frac{8}{45}$ and $\left(\frac{2}{\pi}\right)^{4}$ in the above inequality are the best possible. For more information on this topic, please see [4,5,19], [[14], pp. 38-40, Section 8] and closely related references therein.

Recently the inequalities (1.3) and (1.6) were respectively refined in [9] as

$$
2+\frac{\sin x}{x}+\frac{\tan x}{x}>\frac{\sin x}{x}+2 \frac{\tan (x / 2)}{x / 2}>\frac{x}{\sin x}+\frac{x}{\tan x}>3
$$

and

$$
\begin{aligned}
& \left(\frac{\sin x}{x}\right)^{2}+\frac{\tan x}{x}>\left(\frac{x}{\sin x}\right)^{2}+\frac{x}{\tan x} \\
& >\frac{\sin x}{x}+\left[\frac{\tan (x / 2)}{x / 2}\right]^{2}>\frac{x}{\sin x}+\left[\frac{x / 2}{\tan (x / 2)}\right]^{2}>2 .
\end{aligned}
$$


The hyperbolic counterparts of the last two inequalities in (1.8) were also given in [9] as follows:

$$
\frac{\sinh x}{x}+\left[\frac{\tanh (x / 2)}{x / 2}\right]^{2}>\frac{x}{\sinh x}+\left[\frac{x / 2}{\tanh (x / 2)}\right]^{2}>2 \text {. }
$$

The aim of this paper is to refine and sharpen some of the above-mentioned Huygens and Wilker type inequalities.

\section{Some Lemmas}

In order to attain our aim, we need several lemmas below.

Lemma 2.1. The Bernoulli numbers $B_{2 n}$ for $n \in \mathbb{N}$ have the property

$$
(-1)^{n-1} B_{2 n}=\left|B_{2 n}\right|,
$$

where the Bernoulli numbers $B_{i}$ for $i \geq 0$ are defined by

$$
\frac{x}{e^{x}-1}=\sum_{i=0}^{\infty} \frac{B_{i}}{n !} x^{i}=1+\frac{x}{2}+\sum_{i=1}^{\infty} B_{2 i} \frac{x^{2 i}}{(2 i) !},|x|<2 \pi .
$$

Proof. In [[2], p. 16 and p. 56], it is listed that for $q \geq 1$

$$
\zeta(2 q)=(-1)^{q-1} \frac{(2 \pi)^{2 q}}{(2 q) !} \frac{B_{2 q}}{2},
$$

where $\zeta$ is the Riemann zeta function defined by

$$
\zeta(s)=\sum_{n=1}^{\infty} \frac{1}{n^{s}}
$$

From (2.2), the formula (2.1) follows.

Lemma 2.2. For $0<|x|<\pi$, we have

$$
\frac{x}{\sin x}=1+\sum_{n=1}^{\infty} \frac{2\left(2^{2 n-1}-1\right)\left|B_{2 n}\right|}{(2 n) !} x^{2 n} .
$$

Proof. This is an easy consequence of combining the equality

$$
\begin{aligned}
& \frac{1}{\sin x}=\csc x \\
& =\frac{1}{\sin x}+\sum_{n=1}^{\infty} \frac{(-1)^{n-1} 2\left(2^{2 n-1}-1\right) B_{2 n}}{(2 n) !} x^{2 n-1},
\end{aligned}
$$

see [[1], p. 75, 4.3.68], with Lemma 2.1.

Lemma 2.3 ([[1], p. 75, 4.3.70]). For $0<|x|<\pi$,

$$
\cot x=\frac{1}{x}-\sum_{n=1}^{\infty} \frac{2^{2 n}\left|B_{2 n}\right|}{(2 n) !} x^{2 n-1}
$$

Lemma 2.4. For $0<|x|<\pi$,

$$
\frac{1}{\sin ^{2} x}=\frac{1}{x^{2}}+\sum_{n=1}^{\infty} \frac{2^{2 n}(2 n-1)\left|B_{2 n}\right|}{(2 n) !} x^{2(n-1)} .
$$

Proof. Since

$$
\frac{1}{\sin ^{2} x}=\csc ^{2} x=-\frac{d}{d x}(\cot x),
$$

the formula (2.7) follows from differentiating (2.6).
Lemma 2.5. For $0<|x|<\pi$,

$$
\frac{\cos x}{\sin ^{2} x}=\frac{1}{x^{2}}-\sum_{n=1}^{\infty} \frac{2(2 n-1)\left(2^{2 n-1}-1\right)\left|B_{2 n}\right|}{(2 n) !} x^{2(n-1)}
$$

Proof. This follows from differentiating on both sides of (2.5) and using (2.1).

Lemma 2.6. For $0<|x|<\pi$,

$$
\begin{aligned}
& \frac{1}{\sin ^{3} x}=\frac{1}{2 x}+\frac{1}{x 3} \\
& +\frac{1}{2} \sum_{n=1}^{\infty} \frac{1}{(2 n-1) !}\left[\begin{array}{l}
\frac{\left(2^{2 n+1}-1\right)\left|B_{2 n+2}\right|}{n+1} \\
+\frac{\left(2^{2 n-1}-1\right)\left|B_{2 n}\right|}{n}
\end{array}\right] x^{2 n-1}
\end{aligned}
$$

and

$$
\frac{\cos x}{\sin ^{3} x}=\frac{1}{x^{3}}-\sum_{n=2}^{\infty} \frac{(2 n-1)(n-1) 2^{2 n}\left|B_{2 n}\right|}{(2 n) !} x^{2 n-3} .
$$

Proof. Combining

$$
\frac{1}{\sin ^{3} x}=\frac{1}{2 \sin x}-\frac{1}{2}\left(\frac{\cos x}{\sin ^{2} x}\right)^{\prime}
$$

with Lemma 2.5, the identity (2.5), and Lemma 2.1 gives (2.9).

The equality (2.10) follows from combination of

$$
\frac{\cos x}{\sin ^{3} x}=-\frac{1}{2}\left(\frac{1}{\sin ^{2} x}\right)^{\prime}
$$

with Lemma 2.4.

Lemma 2.7. Let $f$ and $g$ be continuous on $[a, b]$ and differentiable in $(a, b)$ such that $g^{\prime}(x) \neq 0$ in $(a, b)$. If $\frac{f^{\prime}(x)}{g^{\prime}(x)}$ is increasing (or decreasing) in $(a, b)$, then the functions $\frac{f(x)-f(b)}{g(x)-g(b)}$ and $\frac{f(x)-f(a)}{g(x)-g(a)}$ are also increasing (or decreasing) in $(a, b)$.

The above Lemma 2.7 can be found, for examples, in [[3], p. 292, Lemma 1], [[6], p. 57, Lemma 2.3], [[11], p. 92, Lemma 1], and [[12], p. 161, Lemma 2.3].

Lemma 2.8. Let $a_{k}$ and $b_{k}$ for $k \in \mathbb{N}$ be real numbers and the power series

$$
A(x)=\sum_{k=1}^{\infty} a_{k} x^{k} \text { and } B(x)=\sum_{k=1}^{\infty} b_{k} x^{k}
$$

be convergent on $(-R, R)$ for some $R>0$. If $b_{k}>0$ and the ratio $\frac{a_{k}}{b_{k}}$ is strictly increasing for $k \in \mathbb{N}$, then the function $\frac{A(x)}{B(x)}$ is also strictly increasing on $(0, R)$.

The above Lemma 2.8 can be found, for examples, in [[3], p. 292, Lemma 2], [[15], p. 71, Lemma 1], and [[20], Lemma 2.2]. 


\section{Main Results}

Now we are in a position to state and prove our main results, refinements and sharpening of some Huygens and Wilker type inequalities mentioned in the first section.

Theorem 3.1. For $|x| \in\left(0, \frac{\pi}{2}\right)$, we have

$3+\frac{1}{60} x^{3} \sin x<2 \frac{x}{\sin x}+\frac{x}{\tan x}<3+\frac{8 \pi-24}{\pi^{3}} x^{3} \sin x$.

The scalars $\frac{1}{60}$ and $\frac{8 \pi-24}{\pi^{3}}$ in (3.1) are the best possible.

Proof. Let

$$
f(x)=\frac{\frac{2 x}{\sin x}+\frac{x}{\tan x}-3}{x^{3} \sin x}=\frac{\frac{2 x}{\sin ^{2} x}+\frac{x \cos x}{\sin ^{2} x}-\frac{3}{\sin x}}{x^{3}}
$$

for $x \in\left(0, \frac{\pi}{2}\right)$. By virtue of (2.4), (2.7), and (2.8), we have

$$
\begin{aligned}
& f(x)=\frac{1}{3}\left[\begin{array}{l}
\sum_{n=1}^{\infty} \frac{2^{2 n+1}(2 n-1)}{(2 n) !}\left|B_{2 n}\right| x^{2 n-1} \\
-\sum_{n=1}^{\infty} \frac{\left(2^{2 n}-2\right)}{(2 n) !}\left|B_{2 n}\right| x^{2 n-1} \\
\left.-\sum_{n=1}^{\infty} \frac{\left(2^{2 n}-2\right)(2 n-1)}{(2 n) !}\left|B_{2 n}\right| x^{2 n-1}\right]
\end{array}\right] \\
& =\sum_{n=1}^{\infty} \frac{\left|B_{2 n}\right|}{(2 n) !}\left[\begin{array}{l}
2^{2 n+1}(2 n-1)-(2 n-1)\left(2^{2 n}-2\right) \\
-3\left(2^{2 n}-2\right)
\end{array}\right] x^{2 n-4} \\
& =\sum_{n=2}^{\infty} \frac{(n-2) 2^{2 n+1}+4(n+1)}{(2 n) !}\left|B_{2 n}\right| x^{2 n-4} .
\end{aligned}
$$

So the function $f(x)$ is strictly increasing on $\left(0, \frac{\pi}{2}\right)$. Moreover, it is easy to obtain

$$
\lim _{x \rightarrow 0^{+}} f(x)=\frac{1}{60} \text { and } \lim _{x \rightarrow(\pi / 2)^{-}} f(x)=\frac{8 \pi-24}{\pi^{3}} .
$$

The proof of Theorem 3.1 is complete.

Theorem 3.2. For $0<|x|<\pi / 2$,

$$
\begin{aligned}
& 2+\frac{17}{270} x^{3} \sin x<\frac{x}{\sin x}+\left[\frac{x / 2}{\tan (x / 2)}\right]^{2} \\
& <2+\frac{\pi^{2}+8 \pi-32}{2 \pi^{3}} x^{3} \sin x .
\end{aligned}
$$

The constants $\frac{17}{270}$ and $\frac{\pi^{2}+8 \pi-32}{2 \pi^{3}}$ in (3.2) are the best possible.

Proof. By using (2.4), (2.7), (2.9), and (2.10), the function

$$
\begin{aligned}
& g(x)=\frac{\frac{x}{\sin x}+\left[\frac{x / 2}{\tan (x / 2)}\right]^{2}-2}{x^{3} \sin x} \\
& =\frac{1}{4} \frac{2 x \cos x+4 x \sin x+2 x^{2}-x^{2} \sin ^{2} x-8 \sin ^{2} x}{x^{3} \sin ^{3} x} \\
& =\frac{1}{4 x^{3}}\left(\frac{2 x^{2} \cos x}{\sin ^{3} x}+\frac{4 x}{\sin ^{3} x}+\frac{2 x^{2}}{\sin ^{3} x}-\frac{x^{2}}{\sin x}-\frac{8}{\sin x}\right)
\end{aligned}
$$

may be expanded as

$$
\begin{aligned}
& \left\lceil\sum_{n=1}^{\infty} \frac{2^{2 n+2}(2 n-1)\left|B_{2 n}\right|}{(2 n) !} x^{2 n-1}\right. \\
& -\sum_{n=2}^{\infty} \frac{2^{2 n+1}(2 n-1)(n-1)\left|B_{2 n}\right|}{(2 n) !} x^{2 n-1} \\
& g(x)=\frac{1}{4 x^{3}} \mid \begin{array}{l}
-\sum_{n=2}^{\infty} \frac{\left(2^{2 n}-2\right)(2 n-1)(2 n-2)\left|B_{2 n}\right|}{(2 n) !} x^{2 n-1} \\
+\sum_{n=1}^{\infty} \frac{\left(2^{2 n}-2\right)\left|B_{2 n}\right|}{(2 n) !} x^{2 n+1}
\end{array} \\
& -\sum_{n=1}^{\infty} \frac{\left(2^{2 n}-2\right)\left|B_{2 n}\right|}{(2 n) !} x^{2 n+1} \\
& -\sum_{n=1}^{\infty} \frac{8\left(2^{2 n}-2\right)\left|B_{2 n}\right|}{(2 n) !} x^{2 n-1} \\
& =\frac{1}{4} \sum_{n=2}^{\infty} \frac{\left|B_{2 n}\right|}{(2 n) !}\left[\begin{array}{l}
(2 n-1) 2^{2 n+2}-8\left(2^{2 n}-2\right) \\
-2(2 n-1)(2 n-2)
\end{array}\right] x^{2 n-4} \\
& =\sum_{n=2}^{\infty} \frac{4^{n}(2 n-3)+3+3 n-2 n^{2}}{(2 n) !}\left|B_{2 n}\right| x^{2 n-4} \\
& \triangleq \sum_{n=2}^{\infty} \frac{b_{n}}{(2 n) !}\left|B_{2 n}\right| x^{2 n-4} \text {. }
\end{aligned}
$$

Since $b_{2}=17$ and

$$
\begin{aligned}
& b_{n+1}-b_{n}=4^{n}(6 n-1)-4 n+1 \\
& =(1+3)^{n}(6 n-1)-4 n+1>3 n(6 n-1)-4 n+1 \\
& \quad=18 n(n-2)+29(n-2)+59>0
\end{aligned}
$$

for $n \geq 2$, the sequence $b_{n}$ is increasing and $b_{n} \geq b_{2}=17>0$. Thus, the function $g(x)$ is increasing on $\left(0, \frac{\pi}{2}\right)$. Moreover,

$$
\lim _{x \rightarrow 0^{+}} g(x)=\frac{17}{720}
$$

and

$$
\lim _{x \rightarrow(\pi / 2)^{-}} g(x)=\frac{\pi^{2}+8 \pi-32}{2 \pi^{3}} .
$$

The proof of Theorem 3.2 is complete.

Theorem 3.3. For $x>0$, we have

$$
2 \frac{\sinh x}{x}+\frac{\tanh x}{x}>3+\frac{3}{20} x^{3} \tanh x .
$$


The constant $\frac{3}{20}$ is the best possible.

Proof. Let

$F(x)=\frac{2 \frac{\sinh x}{x}+\frac{\tanh x}{x}-3}{x^{3} \tanh x}=\frac{\sinh 2 x+\sinh x-3 x \cosh x}{x^{4} \sinh x}$ and let

$$
f(x)=\sinh 2 x+\sinh x-3 x \cosh x
$$

and

$$
g(x)=x^{4} \sinh x .
$$

From the power series expansions

$$
\sinh x=\sum_{n=0}^{\infty} \frac{x^{2 n+1}}{(2 n+1) !}
$$

and

$$
\cosh x=\sum_{n=0}^{\infty} \frac{x^{2 n}}{(2 n) !}
$$

it follows that

$$
\begin{aligned}
f^{\prime}(x) & =2 \cosh 2 x-2 \cosh x-2 x \sinh x \\
& =\sum_{n=0}^{\infty} \frac{x^{2 n+1} x^{2 n}}{(2 n) !}-\sum_{n=0}^{\infty} \frac{2 x^{2 n}}{(2 n) !}-\sum_{n=0}^{\infty} \frac{3 x^{2 n+2}}{(2 n+1) !} \\
& =\sum_{n=0}^{\infty} \frac{\left(x^{2 n+1}-2\right) x^{2 n}}{(2 n) !}-\sum_{n=0}^{\infty} \frac{3 x^{2 n+2}}{(2 n+1) !} \\
& =\sum_{n=0}^{\infty} \frac{\left(x^{2 n+1}-2\right) x^{2 n}}{(2 n) !}-\sum_{n=0}^{\infty} \frac{6 n x^{2 n+2}}{(2 n) !} \\
& =\sum_{n=0}^{\infty} \frac{\left(x^{2 n+1}-6 n-2\right) x^{2 n}}{(2 n) !} \\
& \triangleq \sum_{n=0}^{\infty} a_{n} x^{2 n}
\end{aligned}
$$

and

$$
\begin{aligned}
g^{\prime}(x) & =4 x^{3} \sinh x+x^{4} \cosh x \\
& =\sum_{n=0}^{\infty} \frac{4 x^{2 n+4}}{(2 n+1) !}+\sum_{n=0}^{\infty} \frac{4 x^{2 n+4}}{(2 n) !} \\
& =\sum_{n=0}^{\infty} \frac{(2 n+5) x^{2 n+4}}{(2 n+1) !}=\sum_{n=0}^{\infty} \frac{(2 n+3) x^{2 n+2}}{(2 n-1) !} \\
& =\sum_{n=0}^{\infty} \frac{4 n(n-1)\left(4 n^{2}-1\right) x^{2 n}}{(2 n) !} \triangleq \sum_{n=0}^{\infty} b_{n} x^{2 n} .
\end{aligned}
$$

It is easy to see that the quotient

$$
c_{n}=\frac{a_{n}}{b_{n}}=\frac{2^{2 n+1}-6 n-2}{4 n(n-1)\left(4 n^{2}-1\right)}
$$

satisfies

$$
c_{n+1}-c_{n}=\frac{\left(6 n^{2}-17 n+1\right) 4^{n}+18 n^{2}+23 n-1}{2 n^{2}(2 n+3)\left(4 n^{2}-1\right)\left(n^{2}-1\right)}>0
$$

for $n \geq 0$. This means that the sequence $c_{n}$ is increasing. By Lemma 2.8, the function $G(x)=\frac{f^{\prime}(x)}{g^{\prime}(x)}$ is increasing on $(0, \infty)$, and so, by Lemma 2.7 , the function $F(x)=\frac{f(x)}{g(x)}=\frac{f(x)-f(0)}{g(x)-g(0)}$ is increasing on $(0, \infty)$. Moreover, it is not difficult to obtain $\lim _{x \rightarrow 0^{+}} F(x)=c_{2}=\frac{3}{20}$. Theorem 3.3 is thus proved.

Theorem 3.4. For $x>0$,

$$
\frac{\sinh x}{x}+\left[\frac{\tanh (x / 2)}{x / 2}\right]^{2}>2+\frac{23}{720} x^{3} \tanh x .
$$

The number $\frac{23}{720}$ in (3.6) is the best possible.

Proof. Let

$$
\begin{aligned}
F(x) & =\frac{\frac{\sinh x}{x}+\left[\frac{\tanh (x / 2)}{x / 2}\right]^{2}-2}{x^{3} \tanh x} \\
& =\frac{\cosh x}{x^{5} \sinh x(1+\cosh x)}\left[\left(\begin{array}{l}
x \sinh x \cosh x+x \sinh x \\
+4 \cosh x-2 x^{2} \cosh x \\
-4-2 x^{2}
\end{array}\right)\right]
\end{aligned}
$$

and let

$$
f(x)=\cosh x\left(\begin{array}{l}
x \sinh x \cosh x+x \sinh x \\
+4 \cosh x-2 x^{2} \cosh x-4-2 x^{2}
\end{array}\right)
$$

and

$$
g(x)=x^{5} \sinh x(1+\cosh x) .
$$

By the power series expansions in (3.4), we obtain

$$
\begin{aligned}
f(x)= & x \sinh x+\frac{1}{4} \sinh x-\frac{3}{4} x \sinh x \\
& +\frac{1}{2} \sinh (2 x)-4 \cosh x+2 \cosh (2 x) \\
& -x^{2} \cosh (2 x)-2 x^{2} \cosh x+2-x^{2} \\
= & \sum_{n=0}^{\infty} \frac{\frac{3^{2 n+1}}{4}-n 2^{2 n+1}-4 n-\frac{7}{4}}{(2 n+1) !} x^{2 n+2} \\
& +\sum_{n=0}^{\infty} \frac{2^{2 n+1}-4}{(2 n) !} x^{2 n}+2-x^{2} \\
= & \sum_{n=1}^{\infty} \frac{3^{2 n+1}-n 2^{2 n+1}-4 n-\frac{7}{4}}{(2 n+1) !} x^{2 n+2} \\
& +\sum_{n=2}^{\infty} \frac{2^{2 n+1}-4}{(2 n) !} x^{2 n}
\end{aligned}
$$




$$
\begin{aligned}
= & \sum_{n=2}^{\infty} \frac{\frac{3^{2 n-1}}{4}-(n-1) 2^{2 n-1}-4 n+\frac{9}{4}}{(2 n-1) !} x^{2 n} \\
& +\sum_{n=2}^{\infty} \frac{2^{2 n+1}-4}{(2 n) !} x^{2 n} \\
= & \sum_{n=3}^{\infty} \frac{1}{(2 n) !}\left\{n\left[\begin{array}{l}
\frac{3^{2 n-1}}{2}-(n-1) 2^{2 n} \\
-8 n+\frac{9}{2}
\end{array}\right]+2^{2 n+1}-4\right\} x^{2 n} \\
\triangleq & \sum_{n=3}^{\infty} a_{n} x^{2 n}
\end{aligned}
$$

and

$$
\begin{aligned}
g(x) & =x^{5}\left[\frac{1}{2} \sinh (2 x)+\sinh x\right] \\
& =\sum_{n=0}^{\infty} \frac{1}{(2 n) !}\left\{\begin{array}{l}
\left(1+2^{2 n-6}\right)(2 n-4)(2 n-3) \\
\times(2 n-2)(2 n-1) 2 n
\end{array}\right\} x^{2 n} \\
& \triangleq \sum_{n=0}^{\infty} b_{n} x^{2 n}
\end{aligned}
$$

The ratio

$$
\begin{aligned}
& c_{n}=\frac{a_{n}}{b_{n}} \\
& =\frac{n\left[\frac{3^{2 n-1}}{2}-(n-1) 2^{2 n}-8 n+\frac{9}{2}\right]+2^{2 n+1}-4}{\left(1+2^{2 n-6}\right)(2 n-4)(2 n-3)(2 n-2)(2 n-1) 2 n}
\end{aligned}
$$

satisfies

$$
c_{3}=\frac{23}{720}=0.031 \ldots, c_{4}=\frac{17}{336}=0.050 \ldots,
$$

and

$$
c_{5}=\frac{5099}{85680}=0.059 \ldots
$$

Furthermore, when $n \geq 6$ by a simple computation, we have

$$
c_{n+1}-c_{n}=\left[\begin{array}{l}
f_{1}(n)+f_{2}(n) \\
+f_{3}(n)+f_{4}(n)
\end{array}\right] /\left[\begin{array}{l}
3 n\left(16+4^{n}\right)\left(64+4^{n}\right) \\
(n-2)(2 n-3) \\
\left(4 n^{2}-1\right)\left(n^{2}-1\right)
\end{array}\right],
$$

where

$$
\begin{aligned}
f_{1}(n) & =16^{n}\left(144 n^{3}-24 n^{2}-648 n+240\right) \\
& =16^{n}\left[\begin{array}{l}
144 n(n-6)^{2}+1704 n(n-6) \\
+4392(n-6)+26592
\end{array}\right] \\
& >0, \\
f_{2}(n) & =9^{n}\left(1024 n^{3}-3072 n^{2}-640 n+3456\right) \\
& =9^{n}\left[\begin{array}{l}
1024 n(n-6)^{2}+9216 n(n-6) \\
+128(129 n+27)
\end{array}\right] \\
& >0,
\end{aligned}
$$

$$
\begin{aligned}
f_{4}(n) & =18432 n^{3}+7680 n^{2}-3456 n-15744 \\
= & 18432 n(n-6)^{2}+228864 n(n-6) \\
& +384(1839 n-41) \\
> & 0
\end{aligned}
$$

and

$$
\begin{aligned}
& f_{3}(n)=4^{n}\left[\begin{array}{l}
9^{n}\left(10 n^{3}-57 n^{2}-13 n+54\right) \\
-2016 n^{4}+8622 n^{3}+5541 n^{2} \\
-33327 n+17490
\end{array}\right] \\
& =4^{n}\left\{\begin{array}{l}
9^{n}\left[\begin{array}{l}
10 n(n-6)^{2}+63 n(n-6) \\
+5(n-6)+84
\end{array}\right] \\
-2016 n^{4}+8622 n^{3} \\
+5541 n^{2}-33327 n \\
+17490
\end{array}\right\} \\
& >4^{n}\left[\begin{array}{l}
84(1+8)^{n}-2016 n^{4}+8622 n^{3} \\
+5541 n^{2}-33327 n+17490
\end{array}\right] \\
& >4^{n}\left\{\begin{array}{l}
84\left[\begin{array}{l}
1+8\left(\begin{array}{l}
n \\
1
\end{array}\right)+8^{2}\left(\begin{array}{l}
n \\
2
\end{array}\right) \\
+8^{3}\left(\begin{array}{l}
n \\
3
\end{array}\right)+8^{4}\left(\begin{array}{l}
n \\
4
\end{array}\right)
\end{array}\right] \\
-2016 n^{4}+8622 n^{3} \\
+5541 n^{2}-33327 n \\
+17490
\end{array}\right\} \\
& =17574-107023 n+144421 n^{2} \\
& -70226 n^{3}+12320 n^{4} \\
& =12320(n-6)^{4}+225454(n-6)^{3} \\
& +1541473(n-6)^{2} \\
& +4686101(n-6)+5372496 \\
& >0
\end{aligned}
$$

for $n \geq 6$. Hence, the sequence $c_{n}$ is increasing. By Lemma 2.8 , the function $F(x)$ is increasing. Finally, it is easy to see that $\lim _{x \rightarrow 0^{+}} F(x)=c_{3}=\frac{23}{720}$. The proof of Theorem 3.4 is complete.

Theorem 3.5. For $0<|x|<\pi / 2$, we have

$$
\begin{gathered}
4+\frac{1}{10} x^{3} \sin x<3 \frac{x}{\sin x}+\cos x \\
<4+\frac{12 \pi-32}{\pi^{3}} x^{3} \sin x
\end{gathered}
$$

The numbers $\frac{1}{10}$ and $\frac{12 \pi-32}{\pi^{3}}$ are the best possible.

Proof. Let

$$
f(x)=\frac{3 \frac{x}{\sin x}+\cos x-4}{x^{3} \sin x}=\frac{1}{x^{3}}\left(\frac{3 x}{\sin ^{2} x}+\cot x-\frac{4}{\sin x}\right) \text {. }
$$


By (2.4), (2.6), and (2.7), we have

$$
\begin{aligned}
f(x) & =\frac{1}{x^{3}}\left[\begin{array}{l}
\frac{3}{x}-\sum_{n=1}^{\infty} \frac{3(2 n-1) 2^{2 n}}{(2 n) !}\left|B_{2 n}\right| x^{2 n-1}+\frac{1}{x} \\
-\sum_{n=1}^{\infty} \frac{2^{2 n}}{(2 n) !}\left|B_{2 n}\right| x^{2 n-4}+\frac{4}{x} \\
-\sum_{n=1}^{\infty} \frac{4\left(2^{2 n}-1\right)}{(2 n) !}\left|B_{2 n}\right| x^{2 n-1}
\end{array}\right] \\
= & \sum_{n=1}^{\infty} \frac{3(2 n-1) 2^{2 n}-2^{2 n}-4\left(2^{2 n}-1\right)}{(2 n) !}\left|B_{2 n}\right| x^{2 n-4} \\
= & \sum_{n=1}^{\infty} \frac{3(6 n-8) 2^{2 n}+8}{(2 n) !}\left|B_{2 n}\right| x^{2 n-4} \\
= & \sum_{n=2}^{\infty} \frac{3(6 n-8) 2^{2 n}+8}{(2 n) !}\left|B_{2 n}\right| x^{2 n-4} .
\end{aligned}
$$

This shows that the function $f(x)$ is increasing on $\left(0, \frac{\pi}{2}\right)$. Moreover, it is straightforward to obtain

$$
\lim _{x \rightarrow 0^{+}} f(x)=a_{2}=\frac{1}{10}
$$

and

$$
\lim _{x \rightarrow(\pi / 2)^{-}} f(x)=\frac{12 \pi-32}{\pi^{3}} .
$$

The proof of Theorem 3.5 is complete.

Remark 3.1. This paper is a slightly revised version of the preprint [7].

\section{Acknowledgements}

The first author was partially supported by the Project of Shandong Province Higher Educational Science and Technology Program under grant No. J11LA57, China. The second author was partially supported by the NNSF under Grant No. 11361038, by the Natural Science Foundation Project of Chongqing City under Grant No. CSTC2011JJA00024, by the Research Project of Science and Technology of Chongqing Education Commission under Grant No. KJ120625, and by the Fund of Chongqing Normal University, China under Grant No. 10XLR017 and 2011XLZ07, China.

\section{References}

[1] M. Abramowitz and I. A. Stegun (Eds), Handbook of Mathematical Functions with Formulas, Graphs, and Mathematical Tables, National Bureau of Standards, Applied
Mathematics Series 55, 4th printing, with corrections, Washington, 1965.

[2] G. E. Andrews, R. Askey, and R. Roy, Special Functions Encyclopedia of Mathematics and its Applications 71, Cambridge University Press, Cambridge, 1999.

[3] H. Alzer and S.-L. Qiu, Monotonicity theorems and inequalities for complete elliptic integrals, J. Comput. Appl. Math. 172 (2004), no. 2, 289-312.

[4] B.-N. Guo, W. Li, and F. Qi, Proofs of Wilker's inequalities involving trigonometric functions, The 7th International Conference on Nonlinear Functional Analysis and Applications, Chinju, South Korea, August 6-10, 2001; Inequality Theory and Applications, Volume 3, Yeol Je Cho, Jong Kyu Kim, and Sever S. Dragomir (Eds), Nova Science Publishers, Hauppauge, NY, pp. 109-112.

[5] B.-N. Guo, B.-M. Qiao, F. Qi, and W. Li, On newproofs of Wilker's inequalities involving trigonometric functions, Math. Inequal. Appl. 6 (2003), no. 1, 19-22.

[6] Z.-H. Huo, D.-W. Niu, J. Cao, and F. Qi, A generalization of Jordan's inequality and an application, Hacet. J. Math. Stat. 40 (2011), no. 1, 53-61.

[7] W.-D. Jiang, Q.-M. Luo, and F. Qi. Refinements and sharpening of some Huygens and Wilker type inequalities, available online at http://arxiv.org/abs/1201.6477.

[8] C. Mortici, The natural approach of Wilker-Cusa-Huygens inequalities, Math. Inequal. Appl. 14 (2011), no. 3, 535-541.

[9] E. Neuman, On Wilker and Huygnes type inequalities, Math. Inequal. Appl. 15 (2012), no. 2, 271-279.

[10] E. Neuman and J. S_andor, On some inequalities involving trigonometric and hyperbolic functions with emphasis on the Cusa-Huygens, Wilker, and Huygens inequalities, Math. Inequal. Appl. 13 (2010), no. 4, 715-723.

[11] D.-W. Niu, J. Cao, and F. Qi, Generalizations of Jordan's inequality and concerned relations, Politehn. Univ. Bucharest Sci. Bull. Ser. A Appl. Math. Phys. 72 (2010), no. 3, 85-98.

[12] D.-W. Niu, Z.-H. Huo, J. Cao, and F. Qi, A general refinement of Jordan's inequality and a refinement of L. Yang's inequality, Integral Transforms Spec. Funct. 19 (2008), no. 3, 157-164.

[13] F. Qi, L.-H. Cui, and S.-L. Xu, Some inequalities constructed by Tchebysheff's integral inequality, Math. Inequal. Appl. 2 (1999), no. 4, 517-528.

[14] F. Qi, D.-W. Niu, and B.-N. Guo, Refinements, generalizations, and applications of Jordan's inequality and related problems, $\mathrm{J}$. Inequal. Appl. 2009 (2009), Article ID 271923, 52 pages.

[15] F. Qi and A. Sofo, An alternative and united proof of a double inequality for bounding the arithmeticgeometric mean, Politehn. Univ. Bucharest Sci. Bull. Ser. A Appl. Math. Phys. 71 (2009), no. 3, 69-76.

[16] J. S_andor and M. Bencze, On Huygens' trigonometric inequality, RGMIA Res. Rep. Coll. 8 (2005), no. 3, Art. 14; Available online at http://rgmia. org/v8n3.php.

[17] J. S. Sumner, A. A. Jagers, M. Vowe, and J. Anglesio, Inequalities involving trigonometric functions, Amer. Math. Monthly 98 (1991), no. 3, 264-267.

[18] J. B. Wilker, Problem E 3306, Amer. Math. Monthly 96 (1989), no. 1,55 .

[19] S.-H. Wu and H. M. Srivastava, A further refinement of Wilker's inequality, Integral Transforms Spec. Funct. 19 (2008), no. 10, 757-765.

[20] L. Zhu, Some new Wilker-type inequalities for circular and hyperbolic functions, Abstr. Appl. Anal. 2009 (2009), Article ID 485842, 9 pages. 